\title{
Isomorphism Theorem on Vector Spaces over a Ring ${ }^{1}$
}

\author{
Yuichi Futa \\ Tokyo University of Technology \\ Tokyo, Japan
}

\author{
Yasunari Shidama \\ Shinshu University \\ Nagano, Japan
}

\begin{abstract}
Summary. In this article, we formalize in the Mizar system [1, 4] some properties of vector spaces over a ring. We formally prove the first isomorphism theorem of vector spaces over a ring. We also formalize the product space of vector spaces. $\mathbb{Z}$-modules are useful for lattice problems such as LLL (Lenstra, Lenstra and Lovász) [5] base reduction algorithm and cryptographic systems [6, 2].
\end{abstract}

MSC: 15A03 15A04 03B35

Keywords: isomorphism theorem; vector space

MML identifier: VECTSP12, version: 8.1.06 5.44.1305

\section{Bijective Linear Transformation}

From now on $K, F$ denote rings, $V, W$ denote vector spaces over $K, l$ denotes a linear combination of $V$, and $T$ denotes a linear transformation from $V$ to $W$.

Now we state the propositions:

(1) Let us consider a field $K$, finite dimensional vector spaces $V, W$ over $K$, a subset $A$ of $V$, a basis $B$ of $V$, a linear transformation $T$ from $V$ to $W$, and a linear combination $l$ of $B \backslash A$. Suppose $A$ is a basis of $\operatorname{ker} T$ and $A \subseteq B$. Then $T\left(\sum l\right)=\sum(T @ * l)$.

(2) Let us consider a field $F$, vector spaces $X, Y$ over $F$, a linear transformation $T$ from $X$ to $Y$, and a subset $A$ of $X$. Suppose $T$ is bijective. Then $A$ is a basis of $X$ if and only if $T^{\circ} A$ is a basis of $Y$.

\footnotetext{
${ }^{1}$ This work was supported by JSPS KAKENHI grant number JP15K00183. 
(3) Let us consider a field $F$, vector spaces $X, Y$ over $F$, and a linear transformation $T$ from $X$ to $Y$. Suppose $T$ is bijective. Then $X$ is finite dimensional if and only if $Y$ is finite dimensional.

(4) Let us consider a field $F$, a finite dimensional vector space $X$ over $F$, a vector space $Y$ over $F$, and a linear transformation $T$ from $X$ to $Y$. Suppose $T$ is bijective. Then

(i) $Y$ is finite dimensional, and

(ii) $\operatorname{dim}(X)=\operatorname{dim}(Y)$.

Proof: For every basis $I$ of $X, \operatorname{dim}(Y)=\overline{\bar{I}}$.

(5) Let us consider a field $F$, vector spaces $X, Y$ over $F$, a linear combination $l$ of $X$, and a linear transformation $T$ from $X$ to $Y$. If $T$ is one-to-one, then $T @ l=T @ * l$.

Proof: For every element $y$ of $Y,\left(T^{@} l\right)(y)=\sum \operatorname{CFS}(l, T, y)$.

\section{Properties of Linear Combinations of Modules over a Ring}

Now we state the proposition:

(6) Let us consider a field $K$, a vector space $V$ over $K$, subspaces $W_{1}, W_{2}$ of $V$, a basis $I_{1}$ of $W_{1}$, and a basis $I_{2}$ of $W_{2}$. If $V$ is the direct sum of $W_{1}$ and $W_{2}$, then $I_{1} \cap I_{2}=\emptyset$.

Let us consider a field $K$, a vector space $V$ over $K$, subspaces $W_{1}, W_{2}$ of $V$, a basis $I_{1}$ of $W_{1}$, a basis $I_{2}$ of $W_{2}$, and a subset $I$ of $V$. Now we state the propositions:

(7) Suppose $V$ is the direct sum of $W_{1}$ and $W_{2}$ and $I=I_{1} \cup I_{2}$. Then $\operatorname{Lin}(I)=$ the vector space structure of $V$.

Proof: Reconsider $I_{3}=I_{1}$ as a subset of $V$. Reconsider $I_{4}=I_{2}$ as a subset of $V$. For every vector $x$ of $V, x \in W_{1}+W_{2}$ iff $x \in \operatorname{Lin}\left(I_{3}\right)+\operatorname{Lin}\left(I_{4}\right)$.

(8) If $V$ is the direct sum of $W_{1}$ and $W_{2}$ and $I=I_{1} \cup I_{2}$, then $I$ is linearly independent.

Proof: Consider $l$ being a linear combination of $I$ such that $\sum l=0_{V}$ and the support of $l \neq \emptyset . I_{1} \cap I_{2}=\emptyset$. $I_{1}$ misses $I_{2}$. Reconsider $I_{3}=I_{1}$, $I_{4}=I_{2}$ as a subset of $V$. Consider $l_{1}$ being a linear combination of $I_{3}, l_{2}$ being a linear combination of $I_{4}$ such that $l=l_{1}+l_{2}$. Reconsider $l_{3}=l_{1}$ as a linear combination of $I$. Set $v_{1}=\sum l_{3} . v_{1} \neq 0_{V}$ by [3, (25)].

(9) Let us consider a field $K$, a vector space $V$ over $K$, subspaces $W_{1}, W_{2}$ of $V$, a basis $I_{1}$ of $W_{1}$, and a basis $I_{2}$ of $W_{2}$. If $W_{1} \cap W_{2}=\mathbf{0}_{V}$, then $I_{1} \cup I_{2}$ is a basis of $W_{1}+W_{2}$. 
Proof: Set $I=I_{1} \cup I_{2}$. Reconsider $W=W_{1}+W_{2}$ as a strict subspace of $V$. Reconsider $W_{3}=W_{1}, W_{4}=W_{2}$ as a subspace of $W$. Reconsider $I_{0}=I$ as a subset of $W$. For every object $x, x \in W_{3} \cap W_{4}$ iff $x \in \mathbf{0}_{V}$. For every object $x, x \in W$ iff $x \in W_{3}+W_{4}$. $I_{0}$ is base.

\section{First Isomophism Theorem}

Let us consider a field $K$, a finite dimensional vector space $V$ over $K$, and a subspace $W$ of $V$. Now we state the propositions:

(10) There exists a linear complement $S$ of $W$ and there exists a linear transformation $T$ from $S$ to $V / W$ such that $T$ is bijective and for every vector $v$ of $V$ such that $v \in S$ holds $T(v)=v+W$.

Proof: Set $S=$ the linear complement of $W$. Set $V_{1}=V / W$. Define $\mathcal{P}$ [vector of $V$, vector of $\left.V_{1}\right] \equiv \$_{2}=\$_{1}+W$. Consider $f_{1}$ being a function from the carrier of $V$ into the carrier of $V_{1}$ such that for every vector $v$ of $V, \mathcal{P}\left[v, f_{1}(v)\right]$. Set $T=f_{1} \uparrow($ the carrier of $S)$. For every vector $v$ of $V$ such that $v \in S$ holds $T(v)=v+W$. The carrier of $V_{1} \subseteq \operatorname{rng} T$. For every objects $x_{1}, x_{2}$ such that $x_{1}, x_{2} \in$ the carrier of $S$ and $T\left(x_{1}\right)=T\left(x_{2}\right)$ holds $x_{1}=x_{2}$.

(11) (i) $V / W$ is finite dimensional, and

(ii) $\operatorname{dim}(V / W)+\operatorname{dim}(W)=\operatorname{dim}(V)$.

The theorem is a consequence of (10) and (4).

Let $K$ be a ring, $V, U$ be vector spaces over $K, W$ be a subspace of $V$, and $f$ be a linear transformation from $V$ to $U$. Assume the carrier of $W \subseteq$ the carrier of ker $f$. The functor $f / W$ yielding a linear transformation from $\bar{V} / W$ to $U$ is defined by

(Def. 1) for every vector $A$ of $V / W$ and for every vector $a$ of $V$ such that $A=a+W$ holds it $(A)=f(a)$.

The functor CQFunctional $f$ yielding a linear transformation from $V / \operatorname{ker} f$ to $U$ is defined by the term

(Def. 2) $f / \operatorname{ker} f$.

Observe that CQFunctional $f$ is one-to-one.

Now we state the proposition:

(12) Let us consider a ring $K$, vector spaces $V, U$ over $K$, and a linear transformation $f$ from $V$ to $U$. Then there exists a linear transformation $T$ from $V / \operatorname{ker} f$ to $\operatorname{im} f$ such that

(i) $T=$ CQFunctional $f$, and

(ii) $T$ is bijective. 
Proof: Set $T=$ CQFunctional $f$. For every object $x, x \in \operatorname{rng} T$ iff $x \in$ $\operatorname{rng} f$.

Let $K$ be a ring, $V, U, W$ be vector spaces over $K, f$ be a linear transformation from $V$ to $U$, and $g$ be a linear transformation from $U$ to $W$. One can verify that the functor $g \cdot f$ yields a linear transformation from $V$ to $W$.

\section{The Product Space of Vector Spaces}

Let $K$ be a ring.

A sequence of vector spaces over $K$ is a non empty finite sequence and is defined by

(Def. 3) for every set $S$ such that $S \in \operatorname{rng} i t$ holds $S$ is a vector space over $K$.

Note that every sequence of vector spaces over $K$ is Abelian group yielding.

Let $G$ be a sequence of vector spaces over $K$ and $j$ be an element of $\operatorname{dom} G$. One can check that the functor $G(j)$ yields a vector space over $K$. Let $j$ be an element of $\operatorname{dom} \bar{G}$. One can verify that the functor $G(j)$ yields a vector space over $K$. The functor multop $G$ yielding a multi-operation of the carrier of $K$ and $\bar{G}$ is defined by

(Def. 4) len $i t=\operatorname{len} \bar{G}$ and for every element $j$ of $\operatorname{dom} \bar{G}, i t(j)=$ the left multiplication of $G(j)$.

The functor $\prod G$ yielding a strict, non empty vector space structure over $K$ is defined by the term

(Def. 5) $\left\langle\prod \bar{G}, \Pi^{\circ}\left\langle+_{G_{i}}\right\rangle_{i},\left\langle 0_{G_{i}}\right\rangle_{i}, \Pi^{\circ}\right.$ multop $\left.G\right\rangle$.

Let us note that $\prod G$ is Abelian, add-associative, right zeroed, right complementable, vector distributive, scalar distributive, scalar associative, and scalar unital.

\section{Cartesian Product of Vector Spaces}

From now on $K$ denotes a ring.

Let $K$ be a ring and $G, F$ be non empty vector space structures over $K$. The functor $\operatorname{prodmlt}(G, F)$ yielding a function from (the carrier of $K) \times(($ the carrier of $G) \times($ the carrier of $F)$ ) into (the carrier of $G) \times($ the carrier of $F$ ) is defined by

(Def. 6) for every element $r$ of $K$ and for every vector $g$ of $G$ and for every vector $f$ of $F, i t(r,\langle g, f\rangle)=\langle r \cdot g, r \cdot f\rangle$.

The functor $G \times F$ yielding a strict, non empty vector space structure over $K$ is defined by the term 
(Def. 7$)<($ the carrier of $G) \times($ the carrier of $F), \operatorname{prodadd}(G, F), \operatorname{prodzero}(G, F)$, $\operatorname{prodmlt}(G, F)\rangle$.

Let $G, F$ be Abelian, non empty vector space structures over $K$. Note that $G \times F$ is Abelian.

Let $G, F$ be add-associative, non empty vector space structures over $K$. One can verify that $G \times F$ is add-associative.

Let $G, F$ be right zeroed, non empty vector space structures over $K$. One can verify that $G \times F$ is right zeroed.

Let $G, F$ be right complementable, non empty vector space structures over $K$. One can check that $G \times F$ is right complementable.

Now we state the propositions:

(13) Let us consider non empty vector space structures $G, F$ over $K$. Then

(i) for every set $x, x$ is a vector of $G \times F$ iff there exists a vector $x_{1}$ of $G$ and there exists a vector $x_{2}$ of $F$ such that $x=\left\langle x_{1}, x_{2}\right\rangle$, and

(ii) for every vectors $x, y$ of $G \times F$ and for every vectors $x_{1}, y_{1}$ of $G$ and for every vectors $x_{2}, y_{2}$ of $F$ such that $x=\left\langle x_{1}, x_{2}\right\rangle$ and $y=\left\langle y_{1}, y_{2}\right\rangle$ holds $x+y=\left\langle x_{1}+y_{1}, x_{2}+y_{2}\right\rangle$, and

(iii) $0_{G \times F}=\left\langle 0_{G}, 0_{F}\right\rangle$, and

(iv) for every vector $x$ of $G \times F$ and for every vector $x_{1}$ of $G$ and for every vector $x_{2}$ of $F$ and for every element $a$ of $K$ such that $x=\left\langle x_{1}, x_{2}\right\rangle$ holds $a \cdot x=\left\langle a \cdot x_{1}, a \cdot x_{2}\right\rangle$.

(14) Let us consider add-associative, right zeroed, right complementable, non empty vector space structures $G, F$ over $K$, a vector $x$ of $G \times F$, a vector $x_{1}$ of $G$, and a vector $x_{2}$ of $F$. Suppose $x=\left\langle x_{1}, x_{2}\right\rangle$. Then $-x=\left\langle-x_{1}\right.$, $\left.-x_{2}\right\rangle$.

Let $K$ be a ring and $G, F$ be vector distributive, non empty vector space structures over $K$. Let us note that $G \times F$ is vector distributive.

Let $G, F$ be scalar distributive, non empty vector space structures over $K$. One can check that $G \times F$ is scalar distributive.

Let $G, F$ be scalar associative, non empty vector space structures over $K$. Let us note that $G \times F$ is scalar associative.

Let $G, F$ be scalar unital, non empty vector space structures over $K$. Let us observe that $G \times F$ is scalar unital.

Let $G$ be a vector space over $K$. One can check that the functor $\langle G\rangle$ yields a sequence of vector spaces over $K$. Let $G, F$ be vector spaces over $K$. Let us note that the functor $\langle G, F\rangle$ yields a sequence of vector spaces over $K$. Now we state the proposition: 
(15) Let us consider a vector space $X$ over $K$. Then there exists a function $I$ from $X$ into $\prod\langle X\rangle$ such that

(i) $I$ is one-to-one and onto, and

(ii) for every vector $x$ of $X, I(x)=\langle x\rangle$, and

(iii) for every vectors $v, w$ of $X, I(v+w)=I(v)+I(w)$, and

(iv) for every vector $v$ of $X$ and for every element $r$ of the carrier of $K$, $I(r \cdot v)=r \cdot I(v)$, and

(v) $I\left(0_{X}\right)=0 \prod_{\langle X\rangle}$.

Proof: Set $C_{3}=$ the carrier of $X$. Consider $I$ being a function from $C_{3}$ into $\prod\left\langle C_{3}\right\rangle$ such that $I$ is one-to-one and onto and for every object $x$ such that $x \in C_{3}$ holds $I(x)=\langle x\rangle$. For every vectors $v, w$ of $X, I(v+w)=$ $I(v)+I(w)$. For every vector $v$ of $X$ and for every element $r$ of the carrier of $K, I(r \cdot v)=r \cdot I(v)$.

Let $K$ be a ring and $G, F$ be sequences of vector spaces over $K$. One can verify that the functor $G^{\frown} F$ yields a sequence of vector spaces over $K$. Now we state the propositions:

(16) Let us consider vector spaces $X, Y$ over $K$. Then there exists a function $I$ from $X \times Y$ into $\prod\langle X, Y\rangle$ such that

(i) $I$ is one-to-one and onto, and

(ii) for every vector $x$ of $X$ and for every vector $y$ of $Y, I(x, y)=\langle x, y\rangle$, and

(iii) for every vectors $v, w$ of $X \times Y, I(v+w)=I(v)+I(w)$, and

(iv) for every vector $v$ of $X \times Y$ and for every element $r$ of $K, I(r \cdot v)=$ $r \cdot I(v)$, and

(v) $I\left(0_{X \times Y}\right)=0 \prod_{\langle X, Y\rangle}$.

Proof: Set $C_{3}=$ the carrier of $X$. Set $C_{4}=$ the carrier of $Y$. Consider $I$ being a function from $C_{3} \times C_{4}$ into $\prod\left\langle C_{3}, C_{4}\right\rangle$ such that $I$ is one-to-one and onto and for every objects $x, y$ such that $x \in C_{3}$ and $y \in C_{4}$ holds $I(x, y)=\langle x, y\rangle$. For every vectors $v, w$ of $X \times Y, I(v+w)=I(v)+I(w)$. For every vector $v$ of $X \times Y$ and for every element $r$ of $K, I(r \cdot v)=r \cdot I(v)$.

(17) Let us consider sequences of vector spaces $X, Y$ over $K$. Then there exists a function $I$ from $\prod X \times \prod Y$ into $\prod\left(X^{\frown} Y\right)$ such that

(i) $I$ is one-to-one and onto, and

(ii) for every vector $x$ of $\prod X$ and for every vector $y$ of $\prod Y$, there exist finite sequences $x_{1}, y_{1}$ such that $x=x_{1}$ and $y=y_{1}$ and $I(x, y)=$ $x_{1} \frown y_{1}$, and 
(iii) for every vectors $v, w$ of $\prod X \times \prod Y, I(v+w)=I(v)+I(w)$, and

(iv) for every vector $v$ of $\prod X \times \prod Y$ and for every element $r$ of the carrier of $K, I(r \cdot v)=r \cdot I(v)$, and

(v) $I\left(0 \prod X \times \prod Y\right)=0 \prod(X \frown Y) \cdot$

Proof: Reconsider $C_{1}=\bar{X}, C_{2}=\bar{Y}$ as a non-empty, non empty finite sequence. Consider $I$ being a function from $\prod C_{1} \times \prod C_{2}$ into $\prod\left(C_{1} \frown C_{2}\right)$ such that $I$ is one-to-one and onto and for every finite sequences $x, y$ such that $x \in \prod C_{1}$ and $y \in \prod C_{2}$ holds $I(x, y)=x^{\frown} y$. Set $P_{1}=\prod X$. Set $P_{2}=\prod Y$. For every natural number $k$ such that $k \in \operatorname{dom} \overline{X \frown Y}$ holds $\overline{X \frown Y}(k)=\left(C_{1} \frown C_{2}\right)(k)$. For every vector $x$ of $\prod X$ and for every vector $y$ of $\prod Y$, there exist finite sequences $x_{1}, y_{1}$ such that $x=x_{1}$ and $y=y_{1}$ and $I(x, y)=x_{1} \frown y_{1}$. For every vectors $v, w$ of $P_{1} \times P_{2}, I(v+w)=I(v)+I(w)$. For every vector $v$ of $P_{1} \times P_{2}$ and for every element $r$ of the carrier of $K$, $I(r \cdot v)=r \cdot I(v)$ by [7, $(9)]$.

(18) Let us consider vector spaces $G, F$ over $K$. Then

(i) for every set $x, x$ is a vector of $\prod\langle G, F\rangle$ iff there exists a vector $x_{1}$ of $G$ and there exists a vector $x_{2}$ of $F$ such that $x=\left\langle x_{1}, x_{2}\right\rangle$, and

(ii) for every vectors $x, y$ of $\prod\langle G, F\rangle$ and for every vectors $x_{1}, y_{1}$ of $G$ and for every vectors $x_{2}, y_{2}$ of $F$ such that $x=\left\langle x_{1}, x_{2}\right\rangle$ and $y=\left\langle y_{1}\right.$, $\left.y_{2}\right\rangle$ holds $x+y=\left\langle x_{1}+y_{1}, x_{2}+y_{2}\right\rangle$, and

(iii) ${ }^{0} \prod\langle G, F\rangle=\left\langle 0_{G}, 0_{F}\right\rangle$, and

(iv) for every vector $x$ of $\prod\langle G, F\rangle$ and for every vector $x_{1}$ of $G$ and for every vector $x_{2}$ of $F$ such that $x=\left\langle x_{1}, x_{2}\right\rangle$ holds $-x=\left\langle-x_{1},-x_{2}\right\rangle$, and

(v) for every vector $x$ of $\prod\langle G, F\rangle$ and for every vector $x_{1}$ of $G$ and for every vector $x_{2}$ of $F$ and for every element $a$ of $K$ such that $x=\left\langle x_{1}\right.$, $\left.x_{2}\right\rangle$ holds $a \cdot x=\left\langle a \cdot x_{1}, a \cdot x_{2}\right\rangle$.

Proof: Consider $I$ being a function from $G \times F$ into $\prod\langle G, F\rangle$ such that $I$ is one-to-one and onto and for every vector $x$ of $G$ and for every vector $y$ of $F$, $I(x, y)=\langle x, y\rangle$ and for every vectors $v, w$ of $G \times F, I(v+w)=I(v)+I(w)$ and for every vector $v$ of $G \times F$ and for every element $r$ of $K, I(r \cdot v)=r \cdot I(v)$ and $0_{\prod\langle G, F\rangle}=I\left(0_{G \times F}\right)$. For every set $x, x$ is a vector of $\prod\langle G, F\rangle$ iff there exists a vector $x_{1}$ of $G$ and there exists a vector $x_{2}$ of $F$ such that $x=\left\langle x_{1}\right.$, $\left.x_{2}\right\rangle$. For every vectors $x, y$ of $\prod\langle G, F\rangle$ and for every vectors $x_{1}, y_{1}$ of $G$ and for every vectors $x_{2}, y_{2}$ of $F$ such that $x=\left\langle x_{1}, x_{2}\right\rangle$ and $y=\left\langle y_{1}, y_{2}\right\rangle$ holds $x+y=\left\langle x_{1}+y_{1}, x_{2}+y_{2}\right\rangle \cdot 0_{\prod\langle G, F\rangle}=\left\langle 0_{G}, 0_{F}\right\rangle$. For every vector $x$ of $\prod\langle G, F\rangle$ and for every vector $x_{1}$ of $G$ and for every vector $x_{2}$ of $F$ such 
that $x=\left\langle x_{1}, x_{2}\right\rangle$ holds $-x=\left\langle-x_{1},-x_{2}\right\rangle$. For every vector $x$ of $\prod\langle G, F\rangle$ and for every vector $x_{1}$ of $G$ and for every vector $x_{2}$ of $F$ and for every element $a$ of $K$ such that $x=\left\langle x_{1}, x_{2}\right\rangle$ holds $a \cdot x=\left\langle a \cdot x_{1}, a \cdot x_{2}\right\rangle$.

\section{REFERENCES}

[1] Grzegorz Bancerek, Czesław Byliński, Adam Grabowski, Artur Korniłowicz, Roman Matuszewski, Adam Naumowicz, Karol Pąk, and Josef Urban. Mizar: State-of-the-art and beyond. In Manfred Kerber, Jacques Carette, Cezary Kaliszyk, Florian Rabe, and Volker Sorge, editors, Intelligent Computer Mathematics, volume 9150 of Lecture Notes in Computer Science, pages 261-279. Springer International Publishing, 2015. ISBN 978-3319-20614-1. doi:10.1007/978-3-319-20615-8_17.

[2] Wolfgang Ebeling. Lattices and Codes. Advanced Lectures in Mathematics. Springer Fachmedien Wiesbaden, 2013.

[3] Yuichi Futa, Hiroyuki Okazaki, and Yasunari Shidama. Submodule of free $\mathbb{Z}$-module. Formalized Mathematics, 21(4):273-282, 2013. doi:10.2478/forma-2013-0029.

[4] Adam Grabowski, Artur Korniłowicz, and Adam Naumowicz. Four decades of Mizar. Journal of Automated Reasoning, 55(3):191-198, 2015. doi:10.1007/s10817-015-9345-1

[5] A. K. Lenstra, H. W. Lenstra Jr., and L. Lovász. Factoring polynomials with rational coefficients. Mathematische Annalen, 261(4):515-534, 1982. doi 10.1007/BF01457454

[6] Daniele Micciancio and Shafi Goldwasser. Complexity of lattice problems: a cryptographic perspective. The International Series in Engineering and Computer Science, 2002.

[7] Yasunari Shidama. Differentiable functions on normed linear spaces. Formalized Mathematics, 20(1):31-40, 2012. doi:10.2478/v10037-012-0005-1

Received August 30, 2017

The English version of this volume of Formalized Mathematics was financed under agreement 548/P-DUN/2016 with the funds from the Polish Minister of Science and Higher Education for the dissemination of science. 